\title{
Multiple-event probability in general-relativistic quantum mechanics: a discrete model
}

\author{
Mauricio Mondragon, Alejandro Perez, Carlo Rovelli \\ Centre de Physique Théorique de Luminy*, Université de la Méditerranée, F-13288 Marseille, EU
}

November 5, 2018

\begin{abstract}
We introduce a simple quantum mechanical model in which time and space are discrete and periodic. These features avoid the complications related to continuous-spectrum operators and infinite-norm states. The model provides a tool for discussing the probabilistic interpretation of generally-covariant quantum systems, without the confusion generated by spurious infinities. We use the model to illustrate the formalism of general-relativistic quantum mechanics, and to test the definition of multiple-event probability introduced in a companion paper. We consider a version of the model with unitary time-evolution and a version without unitary time-evolution.
\end{abstract}

PACS number(s): 04.20.Cv, 03.65.Ta, 04.60.Ds, 04.60.Pp

\section{Introduction}

Fundamental physical systems may be governed by a Wheeler-DeWitt-like equation, rather than a Schrödinger equation. For such systems, the conventional probabilistic interpretation of quantum mechanics cannot be directly applied, and the formulation of a consistent probabilistic interpretation is a controversial problem on which there is no consensus yet $1,2,1,4,4,5,6,8,7,4,10$. The complexity of this conceptual problem is increased by specific technical difficulties related to the presence of continuous-spectrum operators, infinite-norm states, gauge-groups having infinite volume, and the like. These technical difficulties can often be resolved by appropriate techniques, such as generalized states, Gelfand triples, group integration, and similar. But the technical difficulties are often muddled with the core conceptual problem of making sense of general-relativistic quantum mechanics, creating a confusing situation. In this paper, we introduce a simple discrete model where all interesting operators are bounded and have discrete spectrum, so that many of these technical difficulties do not appear. The model provides a tool for discussing the probabilistic interpretation of generally-covariant quantum systems, without getting entangled in the confusion generated by spurious infinities and similar complications.

We use the model for illustrating the general-relativistic formulation of quantum mechanics developed in [5, 6] and in particular the prescription for computing multiple-event probability given in the companion paper [1]. In [11, we have argued that multi-event probability can be always re-conducted to single-event probability, provided that the dynamics and the quantum nature of physical measuring

\footnotetext{
${ }^{*}$ Unité mixte de recherche (UMR 6207) du CNRS et des Universitées de Provence (Aix-Marseille I), de la Mediterranée (Aix-Marseille II) et du Sud (Toulon-Var); laboratoire affilié à la FRUMAM (FR 2291).
} 
apparatuses are taken into account. Here we use the discrete model for illustrating and testing this general technique for computing multiple-event probabilities.

We begin by introducing the model in the simpler context in which there is unitary evolution in time (Sec. 22). We discuss its general-relativistic formulation in Sec. 3. The probabilistic interpretation is discussed in in Sec. 4. The interesting "timeless" case, where there is no unitary time-evolution is discussed in Sec. 5. The modification to the formalism required in the continuum case will be discussed elsewhere 12 .

As pointed out in [11, the formalism that we obtain shows an intriguing convergence with the Hartle-Halliwell's history formulation of quantum mechanics 4. This formulation of quantum theory has been largely motivated by the need of understanding the probabilistic interpretation of quantum mechanics in the "timeless" context, and the problems has been discussed in depth; see [13] and references therein. The conceptual difference between the two approaches has been discussed in detail in 11 .

\section{The model}

Consider a particle in a finite region of two-dimensional space-time. For simplicity, assume that space and time are both periodic and the region has the topology of a torus. We shall later take the time period to infinity. Discretize this region by means of a lattice formed by $N=N_{x} \times N_{t}$ points. Denote the lattice points as $s=(x, t)$, where $x=1,2, \ldots, N_{x}$ and $t=1,2, \ldots, N_{t}$. For convenience, we take $x$ (respectively $t$ ) periodic modulo $N_{x}$ (respectively $N_{t}$ ) that is, we identify $x+N_{x}$ with $x$, and $t+N_{t}$ with $t$. We write the space-time quantum mechanical wave function of the system in the form $\psi(x, t)$, where, we insist, $x$ and $t$ are here discrete, integer, variables.

To begin with, assume the dynamics is given by a unitary evolution in $t$. We drop this assumption below in Sec. 5. Thus assume for the moment that there is a unitary $N_{x} \times N_{x}$ matrix $\mathcal{U}$ and the wave functions $\psi(x, t)$ that are consistent with the dynamics of the theory are those satisfying

$$
\psi(x, t+1)=\mathcal{U} \psi(x, t)=\sum_{y=1}^{N_{x}} \mathcal{U}_{x}^{y} \psi(y, t) .
$$

This is a discrete version of the Schrödinger equation: we can intuitively view the relation with a continuous theory as given by $\mathcal{U} \sim e^{-i H_{0} \tau}$, where $H_{0}$ is the conventional non-relativistic hamiltonian and $\tau$ is the lattice spacing in the time direction (we put $\hbar=1$ throughout this work). In other words, $\mathcal{U}$ is unitary-step time-evolution operator, that is, the the operator that advance the state by one lattice step in the time direction. The full time-evolution operator is given by

$$
\mathcal{U}(t)=\mathcal{U}^{t}
$$

Its matrix elements

$$
W\left(x^{\prime}, t^{\prime} ; x, t\right)=\mathcal{U}_{x^{\prime}}{ }^{x}\left(t^{\prime}-t\right)
$$

form the discrete analog of the propagator of the Schrödinger equation. We assume that $\mathcal{U}^{N_{t}}=\mathbb{1}$, in order to respect the periodicity, a condition that disappears in the $N_{t} \rightarrow \infty$ limit in which we are interested. In non-relativistic quantum mechanics, $\mathcal{U}_{x^{\prime}}{ }^{x}\left(t^{\prime}-t\right)$ is interpreted as the probability amplitude of finding the system at the point $x^{\prime}$ at time $t^{\prime}$ if we have found the system at the point $x$ at time $t$.

One may also consider a variant of the model, in which $\mathcal{U}_{x}^{y}$ is non-vanishing only if $|x-y| \leq k$, where, say, $k=1$. This is typically the case when $\mathcal{U}_{x} y$ is obtained from the discretization of a Schrödinger equation, and derivative operators are discretized by first-neighborhood differences. We call this version "micro-local", since it propagates at finite speed on the lattice. (Recall, however, that the nonrelativistic Schrödinger equation propagates at infinite speed.) This micro-locality is in general inconsistent with the unitarity of $\mathcal{U}$. 


\section{General relativistic formalism}

We now illustrate the general-relativistic formulation of the model introduced above. The kinematical state space $\mathcal{K}$ is the linear space formed by all complex functions $\psi(x, t)$, whether or not they satisfy eq. (11). More precisely, we define $\mathcal{K}$ as the Hilbert space $\mathcal{K}=\mathbb{C}^{N_{x} \times N_{t}}$ spanned by the orthonormal basis $|s\rangle=|x, t\rangle$. We write $\psi(x, t)=\langle x, t \mid \psi\rangle=\psi(s)=\langle s \mid \psi\rangle$. The physical state space $\mathcal{H}$ is the linear subspace of $\mathcal{K}$ formed by the states that satisfy the dynamics, namely by the functions $\psi(x, t)$ that satisfy (11). Since these states are uniquely determined by their value on any fixed time slice $t=$ constant, we have that $\mathcal{H} \sim \mathbb{C}^{N_{x}}$. Notice that, because of the simplicity of our model, $\mathcal{H}$ is a proper subspace of $\mathcal{K}$; this is not true in general (see chapter 5 of [6]).

Let us give a more covariant looking form to (1). For this purpose, let us introduce an $N \times N$ matrix $\mathcal{T}$ (recall $N$ is the total number of lattice points) whose only non-vanishing matrix elements are

$$
\mathcal{T}_{x^{\prime} t^{\prime}}{ }^{x t}=\delta_{t^{\prime}}^{t+1} \mathcal{U}_{x^{\prime}}{ }^{x}
$$

Notice that $\mathcal{T}$ is unitary and

$$
\mathcal{T}^{N_{t}}=\mathbb{1}
$$

(here $\mathbb{1}$ is the unity operator over $\mathcal{K}$ ). Then we can write the dynamical law (1) in the covariant form $\mathcal{T} \psi=\psi$, that is

$$
\sum_{s=1}^{N} \mathcal{T}_{s^{\prime}} s \quad \psi(s)=\psi\left(s^{\prime}\right) .
$$

This is the discrete analog of the continuous Wheeler-DeWitt equation (see [11]). In other words, we can intuitively view the relation with the continuous theory as given by $\mathcal{T} \sim e^{-i H \tau} \sim e^{-i\left(p_{t}+H_{0}\right) \tau}$, where $H$ is the relativistic hamiltonian [6], or "hamiltonian constraint" and $\tau$ is a small parameter-time step.

The generalized projector $\mathbb{P}$ that defines the relativistic formalism is defined by

$$
\mathbb{P}=\sum_{n} \mathcal{T}^{n}
$$

where, because of the periodicity $\mathcal{T}^{n+N_{t}}=\mathcal{T}^{n}$, in (7) we sum only over $n=1, \ldots, N_{t}$. The definition (7) can be seen as the discrete version of the expression

$$
\mathbb{P}=\int d \tau e^{-i \tau\left(p_{t}+H_{0}\right)}
$$

which defines the generalized projector in the continuous case (see eq. (5.58) of [6] and eq. (5) of [1]. This expression is also related to the "group averaging" technique, see [14]).

It is easy to check that $\mathbb{P}$ is $N_{t}$ times the projector operator $\tilde{\mathbb{P}}: \mathcal{K} \rightarrow \mathcal{H}$. In fact, if $\psi$ is in $\mathcal{H}$, namely satisfies (6), we have immediately $\mathbb{P} \psi=N_{t} \psi$. On the other hand, since $\mathcal{T}$ is unitary, it is diagonalizable and its eigenvalues have the form $e^{i \alpha}$. The physical Hilbert space $\mathcal{H}$ is given by the eigenspace associate with the eigenvalue $\alpha=0$ : Because of the periodicity (5), $N_{t} \alpha=2 \pi I$ for some integer $I$. Acting on the corresponding eigenstate, $\Phi_{\alpha}$

$$
\mathbb{P} \Phi_{\alpha}=\sum_{n} \mathcal{T}^{n} \Phi_{\alpha}=\sum_{n} e^{i n \alpha} \Phi_{\alpha}=\sum_{n} e^{i n 2 \pi I / N_{t}} \Phi_{\alpha}=0, \forall \alpha \neq 0 \quad \text { QED. }
$$

It is important to notice that while the projector $\tilde{\mathbb{P}}$ is ill-defined in the $N_{t} \rightarrow \infty$ limit, the generalized projector $\mathbb{P}$ remains well defined. It is for this reason that $\mathbb{P}$ remains meaningful also when $\mathcal{H}$ fails to be a proper subspace of $\mathcal{K}$. A straightforward calculation shows also that

$$
\left\langle x^{\prime}, t^{\prime}|\mathbb{P}| x, t\right\rangle=\left(\mathcal{U}^{\left(t^{\prime}-t\right)}\right)_{x^{\prime}}{ }^{x}=\left(\mathcal{U}\left(t^{\prime}-t\right)\right)_{x^{\prime}}{ }^{x}=\mathcal{U}_{x^{\prime}}{ }^{x}\left(t^{\prime}-t\right)
$$


that is: the matrix elements of $\mathbb{P}$ are the matrix elements of the evolution operator or, what is the same, the propagator (cf. eq. (3) ).

Notice that we can write

$$
\left\langle x^{\prime}, t^{\prime}|\mathbb{P}| x, t\right\rangle=\left(\mathcal{U}^{\left(t^{\prime}-t\right)}\right)_{x^{\prime}}{ }^{x}=\sum_{x_{1} \ldots x_{t^{\prime}-t-1}} \mathcal{U}_{x^{\prime}}{ }^{x_{1}} \mathcal{U}_{x_{1}}{ }^{x_{2}} \cdots \mathcal{U}_{x_{t^{\prime}-t-1}}{ }^{x} \equiv \sum_{\gamma_{(x, t) \Rightarrow\left(x^{\prime}, t^{\prime}\right)}} U[\gamma]
$$

where the sum is over all the "histories" (sequences of lattice points) $\gamma=\left\{\left(x_{1}, t\right),\left(x_{2}, t+1\right), \ldots,\left(x^{\prime}, t^{\prime}\right)\right\}$ that start at the point $(x, t)$, increase monotonically in time by one time unit at every step, and end at the point $\left(x^{\prime}, t^{\prime}\right)$. This expression illustrates the connection with the sum-over-history formalism (see [4] and references therein). It is important to observe that we can equivalently write

$$
\left\langle s^{\prime}|\mathbb{P}| s\right\rangle=\sum_{s, s_{1}, s_{2}, \ldots, s_{n}} \mathcal{T}_{s}^{s_{1}} \mathcal{T}_{s_{1}}{ }^{s_{2}} \ldots \mathcal{T}_{s_{n}}{ }^{s^{\prime}} \equiv \sum_{\gamma_{s \Rightarrow s^{\prime}}} U[\gamma]
$$

where now the sum can be extended over all possible histories. In fact, because of the form (44) of the matrix $\mathcal{T}$, the only histories that have nonvanishing amplitude are the ones that increase monotonically in time by one time unit at every step. Using this language, the fact that the histories summed over increase monotonically in $t$ is not an intrinsic property of the formalism, but only a property of the matrix $\mathcal{T}_{s^{\prime}} s$. This is the property that we will drop in Sec. 5 .

This history language becomes particularly transparent in the micro-local case, in which the transfer matrix $\mathcal{U}$ is directly given by a (non-unitary) discretization of the Schrödinger equation. Say, for instance, that $H_{0}=\frac{1}{2 m} p^{2}+V(x)$, then, approximating the derivative with finite differences, we write the Schrödinger equation (11) in the form

$$
\psi(x, t+1)=\alpha \psi(x+1, t)+\alpha \psi(x-1, t)+\beta(x) \psi(x, t)
$$

(if $a$ and $\tau$ are the spatial and temporal the lattice-spaces, $\alpha=\frac{i \hbar \tau}{2 m a^{2}}$ and $\beta(x)=1-\frac{i \tau}{\hbar}\left(\frac{\hbar^{2} m}{a^{2}}+V(x)\right)$ ). Then the only non-vanishing matrix elements of the $\mathcal{T}$ matrix are

$$
\mathcal{T}_{x^{\prime} t^{\prime}} x t=\delta_{t^{\prime}}^{t+1}\left(\alpha\left(\delta_{x^{\prime}}^{x+1}+\delta_{x^{\prime}}^{x-1}\right)+\beta(x) \delta_{x^{\prime}}^{x}\right) .
$$

That is, $\mathcal{T}$ has non-vanishing elements only for histories that are "continuous" on the lattice, namely formed by sequences of adjacent (or diagonal) lattice sites.

Let us now come to an important point. For the moment, we have introduced $\mathcal{H}$ as a linear space, without saying anything about its Hilbert-space structure, namely about its scalar product. As a proper subspace of $\mathcal{K}$, the space $\mathcal{H}$ inherits a scalar product from $\mathcal{K}$. However, it is far more convenient to define a different scalar product in $\mathcal{H}$, defined as the scalar product in $\mathcal{K}$, divided by $N_{t}$. That is, if $\psi, \psi^{\prime} \in \mathcal{H}$, we define

$$
\left\langle\psi^{\prime} \mid \psi\right\rangle_{\mathcal{H}} \equiv \frac{1}{N_{t}}\left\langle\psi^{\prime} \mid \psi\right\rangle_{\mathcal{K}}
$$

This definition follows easily from the general definition of the scalar product of a physical space (eq. (5.64) and following lines in [6], and eq. (4) in [11])

$$
\left\langle s^{\prime} \mid s\right\rangle_{\mathcal{H}} \equiv\left\langle\mathbb{P} s^{\prime} \mid \mathbb{P} s\right\rangle_{\mathcal{H}} \equiv\left\langle s^{\prime}|\mathbb{P}| s\right\rangle_{\mathcal{K}}
$$

Thus, we promote $\mathcal{H}$ to an Hilbert space by defining its scalar product by (15). The advantage of this, of course, is that in the limit $N_{t} \rightarrow \infty$ the scalar product $\langle\mid\rangle_{\mathcal{H}}$ remains well defined. In other words, this Hilbert structure is well defined even when $\mathcal{H}$ is not a proper subspace of $\mathcal{K}$. Notice that it follows from this definition that $|x, t\rangle$ is a normalized vector in $\mathcal{K}$ and also $\mathbb{P}|x, t\rangle$ is a normalized vector in $\mathcal{H}$.

From now on, we take $N_{t} \rightarrow \infty$. We interpret this limit as describing the infinite time evolution, not the continuous limit of the lattice spacing. 


\section{Probabilistic interpretation}

According to the basic postulate of relativistic quantum mechanics [] , states in $\mathcal{K}$ represent possible observations. In particular, the state $|x, t\rangle \in \mathcal{K}$ represents the event $(x, t)$ in spacetime: say "the particle is detected by an apparatus" at the spacetime point $(x, t)$. Then (see eqs. (5.59)-(5.60) in [6], or eq. (7) in [11]) the probability amplitude for observing the event $\left(x^{\prime}, t^{\prime}\right)$ if the event $(x, t)$ was detected, is given by

$$
\mathcal{A}_{(x, t) \Rightarrow\left(x^{\prime}, t^{\prime}\right)}=\left\langle x^{\prime}, t^{\prime}|\mathbb{P}| x, t\right\rangle .
$$

By equations (3) and (10), this is the conventional amplitude of standard quantum mechanics, that is, the propagator. The interest of this formulation, on the other hand, is that it remains meaningful and well defined when the dynamics is not unitary in time.

The above definition of probability amplitude is sufficient to define the probability of single events, described by individual states in $\mathcal{K}$. The problem left open in [6], and addressed in [11, was to define the probability for multiple events, or for a subspace of $\mathcal{K}$ with dimension larger than 1 . This cannot be avoided, in particular, when dealing with operators with continuous spectrum, because physical apparatuses have always finite resolution and therefore in general cannot detect individual events. Let us therefore move to multiple-event probabilities.

A case that does not raise difficulties is to define the probability that the particle be detected at $(x, t)$ OR $(y, t)$, where $x \neq y$, but, notice, the $t$ of the two events is the same. In this case, the two events are orthogonal in $\mathcal{H}$, because

$$
\langle y, t|\mathbb{P}| x, t\rangle=(\mathcal{U}(0))_{y}{ }^{x}=\delta_{y}{ }^{x} .
$$

We are therefore in the simple case (i) of Sec. III of [11, where, following [11, we postulate that probabilities add. This is of course nothing but the standard assumptions in non-relativistic quantum mechanics demanding that the probability of being at any one of two different points at the same time is the sum of the two probabilities of being at the individual points. Let us move on to the more interesting case.

What is the probability that the particle be detected at $\left(y, t_{1}\right)$ OR $\left(y, t_{2}\right)$, where, say, $t_{1}<t_{2}$ ? For simplicity, let us focus, instead, on the probability for the particle to be detected in $\left(y, t_{1}\right)$ AND $\left(y, t_{2}\right)$; the two are easily related (see eq. (14) in [11]). Now we have

$$
\left\langle y, t_{2}|\mathbb{P}| y, t_{1}\right\rangle=\mathcal{U}_{y}{ }^{y}\left(t_{2}-t_{1}\right)
$$

which in general does not vanish. This is case (ii) of Sec. III of [11. According to [11, this probability can be given a physical meaning only by explicitly coupling the system to an apparatus that interacts with the system at time $t_{1}$ at the point $y$, namely at the space-time point $s_{1}=\left(y, t_{1}\right)$. Let us therefore do so.

We model the apparatus in the simplest possible form. Introduce a two-state system $|A\rangle$ with $A=Y e s, N o$, and couple it to our system. The kinematical state of the combined system is $\mathcal{K}_{\text {ext }}=$ $\mathbb{C}^{N_{x} \times N_{t}} \otimes \mathbb{C}^{2}$, spanned by the orthonormal basis $|\sigma\rangle=|x, t, A\rangle=|s, A\rangle$. We define the dynamics by replacing the operator $\mathcal{T}$ with the operator $\mathcal{T}_{s_{1}}=\mathcal{T}_{x_{1} t_{1}}$ defined by

$$
\left(\mathcal{T}_{s_{1}}\right)_{\sigma^{\prime}}{ }^{\sigma}=\mathcal{T}_{s^{\prime} A^{\prime}}{ }^{s A}=\mathcal{T}_{s^{\prime}}{ }^{s} \delta_{A^{\prime}}^{A}+\delta_{s_{1}}^{s} \mathcal{T}_{s^{\prime}}{ }^{s}\left(\mathcal{I}_{A^{\prime}}{ }^{A}-\delta_{A^{\prime}}^{A}\right) .
$$

The first term is the free evolution of the system and the free (trivial) evolution of the apparatus. The second term is the interaction. It is different from zero only at the space-time point $s_{1}=\left(x_{1}, t_{1}\right)$, that is, the apparatus flips, if and only if the particle pass through $x=x_{1}$ at time $t=t_{1}$. Here, the evolution of the apparatus is given, instead than the trivial evolution operator $\delta_{A^{\prime}}^{A}$, by the matrix

$$
\mathcal{I}_{A^{\prime}}{ }^{A} \equiv\left(\begin{array}{cc}
0 & 1 \\
1 & 0
\end{array}\right)
$$


which flips the state of the apparatus. We call $\mathbb{P}_{s_{1}}=\mathbb{P}_{x_{1}, t_{1}}$ the generalized propagator defined by this coupling, namely by inserting the operator $\mathcal{T}_{x_{1} t_{1}}$ defined in (20) into (12). That is

$$
\left\langle s^{\prime}\left|\mathbb{P}_{s_{1}}\right| s\right\rangle=\sum_{\tilde{s}_{1}, \tilde{s}_{2}, \ldots, \tilde{s}_{n}}\left(\mathcal{T}_{s_{1}}\right)_{s} \tilde{s}_{1}\left(\mathcal{T}_{s_{1}}\right)_{\tilde{s}_{1}} \tilde{s}_{2} \cdots\left(\mathcal{T}_{s_{1}}\right)_{\tilde{s}_{n}} s^{\prime} \equiv \sum_{\gamma_{s \Rightarrow s^{\prime}}} U_{s_{1}}[\gamma]
$$

It is easy to see that this implies

$$
\left\langle x^{\prime}, t^{\prime}, A^{\prime}\left|\mathbb{P}_{x_{1}, t_{1}}\right| x, t, A\right\rangle=\mathcal{U}_{x^{\prime}}{ }^{x}\left(t^{\prime}-t\right) \delta_{A^{\prime} A}
$$

if $t$ and $t^{\prime}$ are both smaller or larger than $t_{1}$ (the interaction time), or if $t^{\prime}<t_{1}<t$; and

$$
\left\langle x^{\prime}, t^{\prime}, A^{\prime}\left|\mathbb{P}_{x_{1}, t_{1}}\right| x, t, A\right\rangle=\sum_{x^{\prime \prime}} \mathcal{U}_{x^{\prime}} x^{\prime \prime}\left(t^{\prime}-t_{1}\right)\left(\delta_{A^{\prime} A}+\delta_{x^{\prime \prime}}^{x_{1}}\left(\mathcal{I}_{A^{\prime} A}-\delta_{A^{\prime} A}\right)\right) \mathcal{U}_{x^{\prime \prime}}{ }^{x}\left(t_{1}-t\right)
$$

if $t<t_{1}<t^{\prime}$. Notice that this interaction is designed for the apparatus to flip state if and only if the particle is at $x_{1}$ at time $t_{1}$. Using (11), this can be rewritten in a more covariant looking form

$$
\left\langle x^{\prime}, t^{\prime}, A^{\prime}\left|\mathbb{P}_{x_{1}, t_{1}}\right| x, t, A\right\rangle=\sum_{\gamma_{(x, t) \Rightarrow\left(x^{\prime}, t^{\prime}\right)}} U[\gamma]\left(\delta_{A A^{\prime}}+\delta_{\left(x_{1}, t_{1}\right)}^{\gamma}\left(\mathcal{I}_{A A^{\prime}}-\delta_{A A^{\prime}}\right)\right)
$$

where $\delta_{\left(x_{1}, t_{1}\right)}^{\gamma}=1$ if the history goes through $x_{1}, t_{1}$ and $\delta_{\left(x_{1}, t_{1}\right)}^{\gamma}=0$ otherwise.

The multiple probability amplitude is then given by (according to Sec. IV.C of [11])

$$
\mathcal{A}_{(x, t) \Rightarrow\left(\left(x_{1}, t_{1}\right),\left(x^{\prime}, t^{\prime}\right)\right)}=\left\langle x^{\prime}, t^{\prime}, Y e s\left|\mathbb{P}_{x_{1}, t_{1}}\right| x, t, N o\right\rangle .
$$

A straightforward computation gives the probability (eq. (10) of [11)

$$
\mathcal{P}_{(x, t) \Rightarrow\left(\left(x_{1}, t_{1}\right),\left(x^{\prime}, t^{\prime}\right)\right)}=\left|\mathcal{A}_{(x, t) \Rightarrow\left(\left(x_{1}, t_{1}\right),\left(x^{\prime}, t^{\prime}\right)\right)}\right|^{2}=\left|\mathcal{U}_{x^{\prime}}{ }^{x_{1}}\left(t^{\prime}-t_{1}\right)\right|^{2} \quad\left|\mathcal{U}_{x_{1}}{ }^{x}\left(t_{1}-t\right)\right|^{2} .
$$

The reader can check with a tedious but straightforward calculation, using standard quantum mechanics and the wave function collapse algorithm, that this is the correct probability for this sequence of measurements, namely for detecting the particle at $x^{\prime}$ at time $t^{\prime}$ and at $x_{1}$ at time $t_{1}$ if the particle was initially at $x$ at time $t$ (assuming $t<t_{1}<t^{\prime}$ ).

The coupling to an apparatus can be easily generalized to an arbitrary number of apparatuses. For instance, say we have apparatuses at the space-time points $s_{1}, \ldots, s_{K}$ coupled as follows

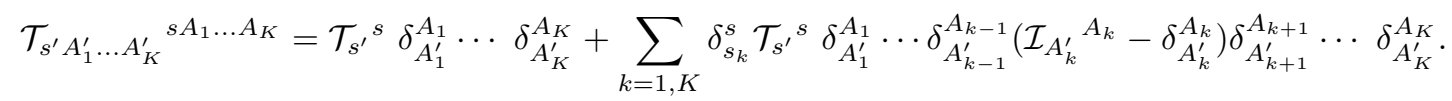

Then the probability to observe the system at the space-time point $\left(x^{\prime}, t^{\prime}\right)$ in conjunction with all apparatuses having detected the particle at all the events $s_{1}, s_{2}, \ldots, s_{K}$, if the system was observed at the space-time point $(x, t)$ in conjunction with all apparatuses being in the No state is

$$
\mathcal{P}_{(x, t) \Rightarrow\left(\left(x_{1}, t_{1}\right) \ldots,\left(x_{K}, t_{K}\right),\left(x^{\prime}, t^{\prime}\right)\right)}=\left|\langle x^{\prime}, t^{\prime}, \underbrace{Y e s, \ldots, Y e s}_{K}\left|\mathbb{P}_{s_{1} \ldots s_{K}}\right| x, t, \underbrace{N o, \ldots, N o}_{\mathrm{K}}\rangle\right|^{2}
$$

The possibility of taking the continuum limit of this expression will be discussed in [12. Again, the reader can check that this is the correct probability for a sequence of measurements, where the wave function is repeatedly collapsed at each measurement.

What we have done so far, indeed, is nothing more than a reformulation of the standard probabilistic interpretation of quantum theory, including the probabilities computed by using the collapse postulate. What is interesting is that this formulation can be readily generalized to the case where there is no unitary evolution, and therefore no evident time-ordering in the system. In fact, the reader 
should notice that the expression (29) does not depend explicitly on the time-ordering of the measurements. Time-ordering is implicitly implemented by the dynamics of the coupled system, which is coded into the form of $\mathcal{T}$. In other words, if $s_{2}$ is earlier in time than $s_{1}$, then the probability (29) becomes automatically the one given by first projecting on $s_{2}$ and then on $s_{1}$. This is particularly transparent in the history language: the histories summed over are only the ones correctly ordered in time because the dynamical operator $\mathcal{T}$ selects only these histories. In this sense, in this formalism the time ordering of the measurements is absorbed into the definition of the dynamics. It is this fact that allows us to generalize the formalism to a genuinely "timeless" situation, namely a situation in which the dynamics is not given by unitary evolution in $t$. This is what we do in the following section.

\section{Extension to the timeless case}

We now consider the extension of the discrete model to the case where there is no unitary evolution in time. We take the same kinematics as the discrete dynamics above, but a different dynamics. That is, we take again a lattice with lattice points $s=(x, t)$, but now there will not be a unitary evolution in $t$. The kinematical Hilbert space is always spanned by the orthonormal basis $|s\rangle=|x, t\rangle$, and we still define the quantum dynamics by the discrete Wheeler-DeWitt equation (6) and by the generalized projector (77).

The novelty is that now we do not assume $\mathcal{T}$ to have the form (4). This form, in fact, is the discrete analog of $H=p_{t}+H_{0}$, which defines the non-relativistic special case of generalized quantum mechanics.

A particularly interesting case is given when $\mathcal{T}$ is still micro-local, that is, has non-vanishing matrix elements $\mathcal{T}_{s^{\prime}}{ }^{s}$ only for lattice points $s=(x, t)$ and $s^{\prime}=\left(x^{\prime}, t\right)$ that differ at most by $k$ lattice steps, namely for $\left|x^{\prime}-x\right|<k$ and $\left|t^{\prime}-t\right|<k$, for instance with $k=1$. This property can follow from instance from a discretization of a continuous relativistic hamiltonian $H$ that has finite degree in the derivatives.

We assume for simplicity that the system is periodic, and there is an integer $Z$ such that $\mathcal{T}^{Z}=\mathbb{1}$. This does not need to be a periodicity in one of the coordinates of the extended configuration space. The example that follows, for instance, has a periodic dynamics. Then the generalized projector is defined by

$$
\mathbb{P}=\sum_{n=1, Z} \mathcal{T}^{n} .
$$

(Otherwise, we have to take the $\operatorname{limit}^{\mathbb{P}}=\lim _{Z \Rightarrow \infty} \frac{1}{Z} \sum_{n=1, Z} \mathcal{T}^{n}$.) All expressions of the previous section continue to hold. In particular, we can still use the coupling (20) to the apparatus system, and define probabilities amplitudes by (26). This shows that multiple probabilities can be defined in general also in a system without unitary time evolution.

The physical interpretation of these probabilities is discussed in detail in the companion paper [11, and will not be repeated here. We only recall here the important fact that in the general case, the probability of detecting the event $s$ is not interpreted as the number of times the system is detected in $s$, instead of being detected in other positions at equal time. Rather, it is interpreted as the number of times the system is detected in $s$, instead of not being detected. For instance, the number of time a detector at $s$ will click, divided the total number of runs of the experiment. The two definitions are equivalent in the non-relativistic case, because in this case if a particle is not at $x$, then the particle must be in another one (and only one other) position of the equal time surface; in the timeless case the first definition becomes meaningless, but the second remain meaningful.

As a concrete example, consider a discretized version of the finite dimensional model studied in [15. In the continuum, this model is defined by a two-dimensional extended configuration space, with coordinates $a$ and $b$, and by the relativistic hamiltonian

$$
H=\frac{1}{2}\left(p_{a}^{2}+p_{b}^{2}+a^{2}+b^{2}\right)-E,
$$


where $E$ is an integer constant. We refer to 15 for all details and motivations. The motions are closed lines in the extended configurations space (in fact, ellipses), and therefore this model does not admit a conventional hamiltonian formulation (because the motion of a hamiltonian system are always monotonic in $t$ and therefore cannot be closed lines). The dynamics of the model is determined by the dynamics of two harmonic oscillators (with $m=\omega=1$ ) with fixed total energy $E_{a}+E_{b}=E$. (The difference is that here the time variable is assumed to be unobservable.) In fact, the two commuting operators $H_{a}=\frac{1}{2}\left(p_{a}^{2}+a^{2}\right)$ and $H_{b}=\frac{1}{2}\left(p_{b}^{2}+b^{2}\right)$ are diagonalized by the states

$$
\Psi_{n_{a} n_{b}}(a, b)=\left\langle a, b \mid n_{a}, n_{b}\right\rangle=H_{n_{a}}(a) H_{n_{b}}(b)
$$

where $n_{a}, n_{b}=0,1, \ldots, \infty$ and $H_{n}(x)$ are the harmonic oscillator eigenstates. Of course:

$$
|a, b\rangle=\sum_{n_{a}=0}^{\infty} \sum_{n_{b}=0}^{\infty} \overline{H_{n_{a}}(a)} \overline{H_{n_{b}}(b)}\left|n_{a}, n_{b}\right\rangle .
$$

The relativistic hamiltonian gives

$$
H\left|n_{a}, n_{b}\right\rangle=\left(E_{a}+E_{b}-E\right)\left|n_{a}, n_{b}\right\rangle=\left(n_{a}+n_{b}+1-E\right)\left|n_{a}, n_{b}\right\rangle .
$$

and defines the physical space via $H \psi=0$. In order for solutions to exist, $E$ must be integer and the physical Hilbert space $\mathcal{H}$ is spanned by the $E$ states $|n, E-1-n\rangle, \quad n=0, \ldots, E-1$. Here $\mathcal{H}$ is a proper subspace of $\mathcal{K}$. The generalized projector is precisely the projector

$$
\mathbb{P}=\frac{1}{2 \pi} \int_{0}^{2 \pi} d \phi e^{-i H \phi}=\sum_{n=0}^{E-1}|n, E-1-n\rangle\langle n, E-1-n| .
$$

Its matrix elements are

$$
\left\langle a^{\prime}, b^{\prime}|\mathbb{P}| a, b\right\rangle=\sum_{n=0}^{E-1} H_{n}\left(a^{\prime}\right) H_{E-1-n}\left(b^{\prime}\right) \overline{H_{n}(a)} \overline{H_{E-1-n}(b)} .
$$

For more details, see 15 .

Let us discretize this model, in order to avoid probability densities and distributions. This can be done as follows. Choose an integer $N \gg E$. Cut-down the kinematical phase space to the space spanned by the states where $\left|n_{a}, n_{b}\right\rangle, \quad n_{a}, n_{b}=0,1, \ldots, N-1$. We write $N=2 M+1$ for later convenience. Introduce the discretized "position states" by analogy with (33), as

$$
|a, b\rangle:=\sum_{n_{a}=0}^{N-1} \sum_{n_{b}=0}^{N-1} \overline{H_{n_{a}}(a)} \overline{H_{n_{b}}(b)}\left|n_{a}, n_{b}\right\rangle
$$

where $a, b=-M \delta,-(M-1) \delta, \ldots, 0, \ldots,(M-1) \delta, M \delta$, and $\delta$ is a suitably chosen small real number 1 Notice that $a$ and $b$ are here discrete variables. The resulting discrete model can be taken as a discrete approximation of the model studied in [15].

\footnotetext{
${ }^{1}$ We want the lattice spacing to satisfy $\delta \ll 1$ in order to be much smaller than the harmonic oscillator vacuum state width $\sqrt{\hbar / m \omega}$, otherwise the vacuum state lattice approximation would be very rough. In the present case $\sqrt{\hbar / m \omega}=1$, since we have chosen units where $\hbar=m=\omega=1$. We also want the lattice spacing to be much smaller than the minimum allowed wavelength: the wavelength is the length on which the state varies; in order for the state to be well approximated by a state on the lattice, the lattice spacing must be much smaller. Since the maximum momentum allowed by the constraint is $p_{\max } \sim \sqrt{2 E}$, this implies that $\delta \ll \frac{2 \pi \hbar}{p_{\max }}$, that is, $\delta \ll \frac{2 \pi}{\sqrt{2 E}}$. Finally, we choose $M$ such that the (finite size) region covered by the lattice is much larger than the maximum amplitude, that is, this region should be large enough to accommodate any state. This implies $M \gg \sqrt{2 E} / \delta$, therefore $N \gg E$. Under these conditions, there are all reasons to believe that the quantum dynamics of the discrete model approximates well the continuum one.
} 
The projector can then be written in the discrete form

$$
\mathbb{P}=\sum_{n=0}^{E-1}|n, E-1-n\rangle\langle n, E-1-n|=\frac{1}{2 N} \sum_{\tau=1}^{2 N} e^{-2 \pi i H \tau / 2 N} .
$$

where $H\left|n_{a}, n_{b}\right\rangle=\left(n_{a}+n_{b}+1-E\right)\left|n_{a}, n_{b}\right\rangle$. (Remember that $2 N$ is the total number of kinematical states.) Equivalently

$$
\mathbb{P}=\frac{1}{2 N} \sum_{\tau=1}^{2 N} \mathcal{T}^{\tau}
$$

where

$$
\mathcal{T}=e^{-2 \pi i H / 2 N}
$$

is the matrix giving the one-step parameter-time evolution in the discretized model. Its matrix elements are

$$
\mathcal{T}_{a^{\prime} b^{\prime}}{ }^{a b}=\left\langle a^{\prime}, b^{\prime}|\mathcal{T}| a, b\right\rangle=\sum_{n_{a}=0}^{N-1} \sum_{n_{b}=0}^{N-1} H_{n_{a}}\left(a^{\prime}\right) H_{n_{b}}\left(b^{\prime}\right) e^{2 \pi i\left(n_{a}+n_{b}+1-E\right) / 2 N} \overline{H_{n_{a}}(a)} \overline{H_{n_{b}}(b)} .
$$

Using this matrix, the matrix elements of the projectors can be written as a sum over histories, as in (12), where, now, $s=(a, b)$ and we sum over all histories long at most $2 N$ steps.

We are now ready to couple the system to an apparatus and calculate multiple-event probabilities. Let's say we have an apparatus that detects the system at the point $\left(a_{1}, b_{1}\right)$. Then we modify $\mathcal{T}$ as follows

$$
\mathcal{T}_{a^{\prime} b^{\prime}}{ }^{a b} \rightarrow\left(\mathcal{T}_{a_{1} b_{1}}\right)_{a^{\prime} b^{\prime} A^{\prime}}{ }^{a b A}=\mathcal{T}_{a^{\prime} b^{\prime}}{ }^{a b} \delta_{A^{\prime}}^{A}+\delta_{a^{1} b^{1}}^{a b} \mathcal{T}_{a^{\prime} b^{\prime}}{ }^{a b}\left(\mathcal{I}_{A^{\prime}}^{A}-\delta_{A^{\prime}}^{A}\right)
$$

This gives the projector

$$
\mathbb{P}_{a_{1} b_{1}}=\sum_{\tau=1}^{2 N}\left(\mathcal{T}_{a_{1} b_{1}}\right)^{\tau}
$$

and the probability amplitude of finding the system at the point $\left(a^{\prime}, b^{\prime}\right)$ of the extended configuration space, with the detector having detected the system in the point $\left(a_{1}, b_{1}\right)$, if the system was detected at the point $(a, b)$ with the detector in the $|N o\rangle$ state, is

$$
\mathcal{A}_{(a, b) \Rightarrow\left(\left(a_{1}, b_{1}\right),\left(a^{\prime}, b^{\prime}\right)\right)}=\left\langle a^{\prime}, b^{\prime}, Y e s\left|\mathbb{P}_{a_{1}, b_{1}}\right| a, b, N o\right\rangle
$$

This is a well-defined multiple-event probability in a system without unitary time evolution. This shows that the prescription of [11] can be implemented, at least in principle, also in a system without unitary time evolution.

We close with a conjecture. It should be interesting to consider the semiclassical limit of these probabilities. In [15], semiclassical coherent states corresponding to classical trajectories (ellipses) were constructed. Consider a semiclassical kinematical wave packet peaked around the point $(a, b)$, and with velocity $\left(p_{a}, p_{b}\right)$. This state is projected by $\mathbb{P}$ onto a a semiclassical state peaked along the corresponding ellipses. In [15, it was shown that the kinematical state space splits into clockwise and anticlockwise moving states. Let $\psi_{a b}^{C}$ and $\psi_{a b}^{A}$ be two such states, belonging to the clockwise and anticlockwise subspace, respectively, both generating a semiclassical motion along a given ellipses (but in opposite directions). Let $(a, b),\left(a^{\prime}, b^{\prime}\right)$ and $\left(a_{1}, b_{1}\right)$ be three points along these ellipses. Consider the two transition amplitudes

$$
\mathcal{A}_{\psi_{a b}^{C} \Rightarrow\left(\left(a_{1}, b_{1}\right),\left(a^{\prime}, b^{\prime}\right)\right)}=\left\langle a^{\prime}, b^{\prime}, Y e s\left|\mathbb{P}_{a_{1}, b_{1}}\right| \psi_{a b}^{C}, N o\right\rangle .
$$

and

$$
\mathcal{A}_{\psi_{a b}^{A} \Rightarrow\left(\left(a_{1}, b_{1}\right),\left(a^{\prime}, b^{\prime}\right)\right)}=\left\langle a^{\prime}, b^{\prime}, Y e s\left|\mathbb{P}_{a_{1}, b_{1}}\right| \psi_{a b}^{A}, N o\right\rangle .
$$

One may expect that in the classical limit the first of these amplitudes is suppressed if $(a, b) \Rightarrow\left(a_{1}, b_{1}\right) \Rightarrow$ $\left(a^{\prime}, b^{\prime}\right)$ is ordered anti-clockwise, and the second is suppressed if $(a, b) \Rightarrow\left(a_{1}, b_{1}\right) \Rightarrow\left(a^{\prime}, b^{\prime}\right)$ is ordered clockwise. 


\section{Conclusion}

Using a simple discrete model, we have shown in detail how multi-event probabilities can be computed in a "timeless" quantum system, governed by a Wheeler-DeWitt-like equation, instead than a Schrödinger equation. We have computed these probabilities using the strategy introduced in [11, namely the observation that multi-event probabilities can be interpreted as probabilities for a single event, if the event includes the presence of a "record" of a feature of the system measured by an apparatus that has interacted with the system itself.

In words, to say that

(i) a particle has been detected at $\left(x_{1}, t_{1}\right)$ and at $\left(x_{2}, t_{2}\right)$ can be interpreted as meaning that we detect a state in which

(ii) the particle is detected at $\left(x_{2}, t_{2}\right)$ and there is a record that the particle has interacted with another system at $\left(x_{1}, t_{1}\right)$.

This interpretation is conceptually satisfying, since how else could we combine events happening at different times, if not by having records? The key difference between (i) and (ii) is of course that in conventional quantum mechanics the property of being at $\left(x_{1}, t_{1}\right)$ and the property of being at $\left(x_{2}, t_{2}\right)$ are represented by quantum operators that do not commute. While the property of being detected at $\left(x_{2}, t_{2}\right)$ and the existence of a record that the particle has interacted with another system at $\left(x_{1}, t_{1}\right)$ are expressed by operators that commute: hence there is a single state where both properties are sharp.

Notice that nothing in the construction presented here assures us that the probabilities computed are independent from the dynamics of the apparatus and its coupling. In fact, in general, the opposite may very well be the case, especially in more complicated situations, or in the continuous case. This is not a defect of the formalism. To the contrary, it is an indication from the theory that physically different apparatuses may give different results (see [13] and references therein). In other words, the philosophy here is not that an abstract apparatus-independent probability has to be well defined. Rather, in any concrete physical situation, where a given apparatus is present, the formalism should be capable of providing the probabilities of the observable outcomes.

\section{Acknowledgments}

This work has been partially completed thanks to the financial support provided by the Programme Alßan, European Union Programme of High Level (scholar-)Grants for Latin America, grant No. E04D033873MX; and by the Programme SFERE-CONACYT (France-Mexico), grant No. 166826.

\section{References}

[1] C Kiefer, Quantum Gravity, (Oxford Science Publications, Oxford 2004).

[2] CJ Isham, "Canonical quantum gravity and the problem of time," Presented at 19th International Colloquium on Group Theoretical Methods in Physics, Salamanca, Spain (1992), 152-288; arXiv:gr-qc/9210011.

[3] KV Kuchar, "Time and interpretations of quantum gravity," in Proceedings of the 4th Canadian Conference on General Relativity and Relativistic Astrophysics, ed. by Winnipeg, World Scientific, Singapore (1992), 211 - 314.

[4] JB Hartle, "Space-time quantum mechanics and the quantum mechanics of space-time," in Gravitation and Quantizations: Proceedings of the 1992 Les Houches Summer School, ed. by B Julia \& J Zinn-Justin, Les Houches Summer School Proceedings v LVII, North Holland, Amsterdam (1995), 285-480; arXiv:gr-qc/9304006. 
[5] C Rovelli, "Partial observables," Phys. Rev. D 65 (2002), 124013; [arXiv: gr-qc/0110035.

C Rovelli, "A note on the foundation of relativistic mechanics. I: Relativistic observables and relativistic states," in Proceedings of the 15th SIGRAV Conference on General Relativity and Gravitational Physics, Rome, September (2002); [arXiv: gr-qc/0111037.

C Rovelli, "A note on the foundation of relativistic mechanics: Covariant hamiltonian general relativity," in Topics in Mathematical Physics, General Relativity and Cosmology in Honor of Jerzy Plebański, edited by H. García-Compeán, B. Mielnik, M. Montesinos, and M. Przanowski, World Scientific, Singapore (2006), pp. 397-407; [arXiv: gr-qc/0202079].

C Rovelli, "Dynamics without time for quantum gravity: Covariant hamiltonian formalism for field theory and Hamilton-Jacobi equation on the space G," in Decoherence and Entropy in Complex Systems. Selected lectures from DICE 2002, ed. by HT Elze, Lecture Notes in Physics 633, Springer, Berlin (2003), 36-62; [arXiv: gr-qc/0207043.

[6] C Rovelli, Quantum gravity, (Cambridge University Press, Cambridge, 2004).

[7] DN Page and WK Wootters, "Evolution without evolution: Dynamics described by stationary observables," Phys. Rev. D27 (1983), 288.

[8] B Dittrich, "Partial and complete observables for Hamiltonian constrained systems," arXiv:gr-qc/0411013.

B Dittrich, "Partial and Complete observables for canonical general relativity," Class. Quant. Grav. 23 (2006), 6155-6184; arXiv:gr-qc/0507106.

[9] CE Dolby, "The conditional probability interpretation of the Hamiltonian constraint," arXiv:gr-qc/0406034.

[10] SJ Olson, JO Dowling, "Information and measurement in covariant quantum theory," [arXiv: quant-ph/0701200.

[11] F Hellmann, M Mondragon, A Perez, C Rovelli "Multiple-event probability in general-relativistic quantum mechanics", Phys. Rev. D 75 (2007), 081301; [arXiv: gr-qc/0610140].

[12] E Alesci, M Mondragon, A Perez, C Rovelli, "Probability in general-relativistic quantum mechanics: continuum models," to appear.

[13] JJ Halliwell, "Somewhere in the universe: Where is the information stored when histories decohere?", Phys. Rev. D 60 (1999), 105031; [arXiv: quant-ph/9902008].

JJ Halliwell, "Trajectories for the wave function of the universe from a simple detector model," Phys. Rev. D 64 (2001), 044008; [arXiv: gr-qc/0008046.

JJ Halliwell, "Decoherent histories for space-time domains," in Time in quantum mechanics, J G Muga, R Sala, I L Egusquiza, Springer, Berlin (2002), 153-182.

[14] D Marolf, "Group averaging and refined algebraic quantization: Where are we now?," arXiv:gr-qc/0011112.

D Giulini, D Marolf, "On the generality of refined algebraic quantization," Class. Quant. Grav. 16 (1999), 2479-2488; [arXiv: gr-qc/9812024.

[15] D Colosi, C Rovelli, "A simple background-independent hamiltonian quantum model," Phys. Rev. D 68 (2003), 104008; arXiv:gr-qc/0306059. 